\title{
DESEMPENHO ECONÔMICO-FINANCEIRO E VALOR DAS AÇÕES EM UM CONTEXTO DE MUDANÇA REGULATÓRIA: O CASO DO SETOR ELÉTRICO BRASILEIRO
}

\section{FINANCIAL PERFORMANCE AND STOCK VALUES IN A CONTEXT OF REGULATORY CHANGE: THE CASE OF THE BRAZILIAN ELECTRIC SECTOR}

\author{
MARCOS LIMA BANDEIRA \\ Controladoria-Geral da União. Endereço: Setor de Autarquias Sul, Quadra 1 \\ Bloco A, | Asa Sul | 70070-905 | Brasília/DF | Brasil. \\ (1) http://orcid.org/0000-0003-1356-2689 \\ mlband2@hotmail.com
}

PAUlO AUGUSTO PETTENUZZO DE BRITTO

Universidade de Brasília. Endereço: Faculdade de Administração, Contabilidade, Economia e Gestão de Políticas Públicas, Campus Darcy Ribeiro, S/N | Asa Norte | 70910-900| Brasília/DF | Brasil.

(D) http://orcid.org/0000-0001-7462-9096

pbritto@unb.br

\section{RESUMO}

O objetivo deste estudo é analisar a relação entre o desempenho econômico-financeiro e o valor das ações das empresas do setor elétrico brasileiro no mercado de capitais, conjuntamente com os reflexos da regulação econômica disposta pela Medida Provisória no 579/2012. O tema analisa aspectos da regulação econômica e do desempenho econômico-financeiro do setor elétrico brasileiro, essencial para o desenvolvimento econômico e social do país. Os achados visam contribuir para o avanço de estudos empíricos que examinam o comportamento do mercado acionário, fundamentado nas informações disponibilizadas pelas empresas e naquelas acessíveis no mercado de firmas de segmentos regulados. Os procedimentos metodológicos envolvem um modelo econométrico de regressão múltipla em painel compreendendo 29 companhias concessionárias e permissionárias de energia elétrica listadas na Bolsa de Valores de São Paulo (B3) entre 2009 e 2016. Em relação ao valor de mercado das ações das companhias do setor elétrico, os resultados apontaram associação negativa com a medida provisória, positiva com o índice Liquidez Corrente e negativa com o Índice de Endividamento. Os resultados indicam que a estratégia de precificação dos investidores em empresas reguladas considera efeitos de intervenções regulatórias juntamente com variáveis típicas de mercado.

Palavras-chave: Regulação econômica. Setor elétrico brasileiro. Mercado de capitais. Desempenho econômico-financeiro. 


\begin{abstract}
The objective of this study is to analyze the degree of relationship between economic-financial performance and the value of the stocks of companies in the Brazilian electric sector in the capital market, together with the effects of the economic regulation provided by Provisional Measure No. 579/2012. The theme of this work can be considered relevant as it analyzes aspects of economic regulation and economic-financial performance of an important segment of the Brazilian economy, essential for the country's economic and social development. The results aim to contribute to the advancement of empirical studies that examine the behavior of the stock market, based on information provided by companies and those available in the market, from firms in regulated segments. The methodological procedures involve a panel econometric regression model comprising 29 concessionaires and permissions electric energy companies listed on the São Paulo Stock Exchange (B3) between 2009 and 2016. Regarding the market value of the shares of the electricity companies, the results indicated a negative association with the provisional measure, positive with the ratio between the Current Liquidity Index and negative with the Indebtedness Ratio. The results indicate that the pricing strategy of investors in regulated companies considers effects of regulatory interventions together with typical market variables.
\end{abstract}

Keywords: Economic regulation. Brazilian electric sector. Capital market. Indicators of financial performance.

\title{
1 INTRODUÇÃO
}

As últimas décadas foram marcadas por ações desenvolvidas pelo estado brasileiro no setor elétrico, com a utilização de variáveis para a redução do custo das tarifas, no intuito de garantir uma trajetória positiva e crescente na instituição de novos modelos para a indústria de energia elétrica.

Em setembro de 2012, foi proposta a edição de Medida Provisória (MP) n ${ }^{\circ}$ 579/2012, posteriormente convertida na Lei $\mathrm{n}^{\mathrm{o}}$ 12.783/2013, que alterou normas da legislação vigente, com objetivos de viabilizar a redução do custo da energia elétrica ao consumidor, de promover a modicidade tarifária, de garantir o suprimento de energia elétrica, bem como de tornar o setor produtivo mais competitivo e de contribuir para o aumento do nível de emprego e renda no Brasil (Ministério de Minas e Energia, Ministério da Fazenda \& Advocacia-Geral da União, 2012).

No entanto, um conjunto de aspectos secundários refletiu negativamente no setor elétrico nacional, com possíveis reflexos no mercado acionário, quando foram evidenciadas desvalorizações de ações das principais empresas de energia elétrica do Brasil. A B3 S.A. registrou, em novembro de 2012, perdas jamais vistas nas últimas duas décadas de companhias do setor elétrico brasileiro, como verificado pelas ações da Centrais Elétricas Brasileiras S.A. (Eletrobras), quando alcançou a segunda maior desvalorização de sua história (Cerqueira, 2013). Dessa forma, os principais impactos identificados com o anúncio dessa medida dizem respeito às estimativas do mercado, com redução no valor das ações das empresas de energia listadas na B3, mesmo aquelas não afetadas diretamente pela ação regulatória (Prado \& Silva, 2013).

Um ano após a implementação da MP no 579/2012, os especialistas avaliaram negativamente a medida, devido à perda de autossuficiência das concessionárias, a contenção e a redução de custos e a readequação de receitas, as dificuldades para manter o equilíbrio econômicofinanceiro das empresas, a suspensão dos investimentos, a diminuição da autossustentabilidade do setor, a limitação na competitividade e as dificuldades com o fluxo de caixa (Godoi, 2013).

A crise do setor provocada pela ação regulatória de 2012 e a ingerência do governo levou a Eletrobras, maior empresa estatal do setor, a acumular prejuízos na ordem de R \$ 13,2 bilhões entre 2012 e 2013, a atrasar pagamentos de fornecedores, a entrar em operações com retornos duvidosos e a perder parte de seu corpo técnico (Batista \& Lima, 2014). 
Em 2017, o governo federal anunciou a pretensão de vender ações da União na Eletrobras, por intermédio da diluição de sua participação na empresa. De acordo com dados das demonstrações financeiras individuais da Eletrobras, a empresa acumula prejuízos contábeis desde o exercício de 2012, com montante registrado de $\mathrm{R} \$ 6,8$ bilhões em 2012, $\mathrm{R} \$ 6,2$ bilhões em 2013, $\mathrm{R}$ \$ 3 bilhões em 2014 e R \$ 14,4 bilhões em 2015. Um plano de redução de custos, implementado pela companhia no segundo trimestre de 2017, teve o intuito de diminuir seu endividamento na ordem de R \$23,4 bilhões (Pamplona \& Wiziack, 2017).

O presente estudo tem como objetivo analisar a relação entre o desempenho econômicofinanceiro, por meio de indicadores, e o valor das ações no mercado acionário de empresas do setor elétrico brasileiro, no contexto da mudança regulatória implementada pela MP n ${ }^{\circ}$ 579/2012.

Este trabalho torna-se relevante ao destacar a necessidade de se investigar os eventuais efeitos sobre o mercado de alterações regulatórias, com o emprego de uma análise multivariada, considerando os efeitos simultâneos de um evento e de variáveis econômico-financeiras sobre o valor de mercado de empresas de um segmento regulado da economia brasileira. Espera-se que os resultados obtidos contribuam para o aprimoramento da regulação do setor, ao comprovar empiricamente que o mercado reage ao conteúdo informacional de intervenções regulatórias juntamente com o de outras variáveis típicas de mercado. Ademais, espera-se que este conhecimento colabore para aperfeiçoar o processo de análise de impacto regulatório por parte dos entes estatais.

O presente artigo está dividido em cinco partes, sendo a primeira esta introdução. Na próxima seção é apresentada a fundamentação teórica, destacando os pressupostos da teoria econômica da regulação, bem como os resultados de estudos sobre a associação entre eventos regulatórios, indicadores econômico-financeiros e mercado de capitais e as hipóteses de pesquisa. A terceira parte discorre sobre os procedimentos metodológicos empregados, incluindo amostra, coleta e fonte de dados, indicadores utilizados e definição do modelo econométrico. A quarta seção traz os resultados da análise empírica, contemplando testes empíricos, análise das estimações e discussão dos resultados encontrados. Por fim, a última seção apresenta as conclusões da pesquisa, as limitações do estudo, as contribuições e as sugestões para trabalhos futuros.

\section{FUNDAMENTAÇÃO TEÓRICA}

Esta seção apresenta, primeiramente, os pressupostos da teoria econômica da regulação, bem como pesquisas envolvendo eventos regulatórios e o mercado de capitais. Em seguida, apresenta os indicadores econômico-financeiros empregados na análise, acompanhado de resenha da literatura que trata de sua associação com o valor de mercado de ações.

\subsection{Teoria Econômica da Regulação}

A regulação é geralmente uma atividade identificável e discreta da atuação estatal, por meio de mecanismos diversos, que pode restringir a conduta dos agentes econômicos, evitar a ocorrência de certas ações comportamentais indesejáveis ou facilitar a condução ordenada da atividade econômica (Baldwin, Cave \& Lodge, 2012). Pohlmann e Alves (2012) ressaltam que a regulação é compreendida como um conjunto de dispositivos legais editado pelo estado ou instituições delegadas com poder coercitivo sobre determinada atividade econômica, área do conhecimento ou setores da economia.

A primeira corrente da regulação sob o enfoque econômico tem caráter normativo. Esta abordagem, baseada no interesse público, defende que a regulação deveria surgir quando da existência de falhas de mercado (existência de bens públicos, monopólio natural, externalidades, mercados imperfeitos, falhas de informação, ocorrência de desemprego e inflação), com o fim de corrigi-las e maximizar o bem-estar social, ou seja, o aspecto normativo explica em que momento 
a regulação deveria surgir e que forma deveria possuir para alcançar o incremento desse bem-estar (Mueller, 2001; Pohlmann \& Alves, 2012).

Diante da dificuldade de definir medidas de bem-estar social, o padrão normativo da teoria do liberalismo econômico, no século passado, disseminou a regulação com a finalidade de corrigir as falhas de mercado (Schymura, 2014). A corrente normativa parte da premissa de que o mercado competitivo perfeito harmoniza, de forma ideal, a busca do interesse particular e a construção do bem coletivo, e sustenta que o papel de regulador, com funções eminentemente técnicas, é ajustar o funcionamento equilibrado do mercado nas relações entre os agentes econômicos (Shymura, 2014).

Laffont e Tirole (1990) salientam que importantes fatores do processo de tomada de decisão dos governos não são discutidos no âmbito normativo da teoria, tampouco aspectos interessantes da ciência política. Nesse sentido, a abordagem normativa incorre em simplificações ao desconsiderar instituições políticas e econômicas e negligencia as questões políticas sobre a economia, no que se refere às enormes restrições sobre a alocação dos recursos pelo sistema político (Laffont, 1994; Mueller, 2001).

Enquanto a corrente da regulação sob o aspecto normativo se centrava nas falhas de mercado e na economia do bem-estar como justificativas para a intervenção do Estado, inflexões críticas em relação ao fenômeno da regulação, fundamentadas na teoria da escolha pública de Buchanan e Tullock (1962), emergiram para elucidar as falhas de governo e os limites dessa intervenção (Salgado, 2003).

Para Buchanan e Tullock (1962), a questão elementar está na análise sobre qualquer motivação estritamente de interesse próprio dos indivíduos e seu comportamento nos processos de escolha social, e no fato de que esses indivíduos, com tal, provavelmente possuem objetivos e propósitos diferentes para os resultados de uma ação coletiva.

Neste contexto, a agenda própria de governos e de organismos internacionais na promoção de programas de ajustes estruturais do Estado é vista, sob a ótica da escolha pública, como uma ingerência política nas transações do mercado, uma vez que ameaça a liberdade individual e o progresso econômico (Borges, 2001).

Outrossim, as trocas no ambiente político estariam na essência do problema econômico, pois, ao considerar a política como um mercado, converge-se para um mecanismo de busca aos objetivos pessoais (Freire, Costa, Gartner \& Clemente, 2013). Para Salgado (2003), a escolha pública preconiza que o interesse público está representado na economia de mercado e qualquer outro ambiente, como o mercado político, apenas confunde os sinais fornecidos pelo sistema de preços e compromete a eficiência desse sistema, assim, o livre mercado é a representação ideal do interesse público.

A teoria econômica da regulação, por seu turno, inovou sob o aspecto descritivo ao propor uma teoria para o processo de escolha dos reguladores sob as hipóteses da relação agente-principal. Com efeito, passou a considerar não somente a oferta de regulação por parte dos reguladores, mas uma demanda por regulação por parte dos agentes privados (Pohlmann \& Alves, 2012).

Nesse sentido, a partir da década de 1970, novos avanços da literatura econômica colocaram em questão a neutralidade do regulador em corrigir as falhas de mercado com premissas do bem-estar social (Schymura, 2014), consolidados nas pesquisas de Stigler (1971), Posner (1974), Peltzman (1976) e Becker (1983).

O estudo de Stigler (1971), pioneiro na formulação de uma teoria da regulação econômica, tem como pontos centrais explicar quem receberá o benefício da regulação ou sobre quem recairá o ônus da regulação, que forma tomará e quais seus efeitos sobre a alocação dos recursos. A regulação pode ser perseguida por uma firma ou imposta por ela. A tese defendida é de que a regulação, em regra, é adquirida pela indústria e fundamentalmente elaborada para seu benefício. O problema da regulação reside em descobrir quando e por que uma indústria se utiliza do Estado para seus propósitos ou se é usada pelo Estado em benefício de terceiros.

Posner (1974) critica as premissas das teorias de regulação econômica na medida em que 
pensa simplificar a oferta de regulação como iniciativa do próprio regulador. Desta forma, propõe um resgate da teoria do interesse público, criada em resposta a uma demanda da sociedade para corrigir práticas de mercado ineficientes, combinando-a com a teoria da captura, formulada em resposta a grupos de interesse que disputam entre si a maximização dos benefícios privados.

Já Peltzman (1976) argumenta que as agências reguladoras não visam beneficiar exclusivamente um único agente econômico, mas, sim, grupos de interesses distintos, sejam produtores ou consumidores. Desta forma, a regulação seria continuamente alterada em resposta a choques exógenos que afetem condutas e desempenho de entes regulados.

Por fim, Becker (1983) parte de um entendimento mais amplo de regulação, considerando estrutura tributária, subsídios e outros instrumentos administrativos como formas de regulação também utilizadas para aumentar o bem-estar de grupos de pressão mais influentes. Desta forma, grupos de pressão menores, mais bem organizados e com preferências coesas tenderiam a obter vantagens por meio da estrutura regulatória.

Diante deste arcabouço, é plausível supor que o mercado compreenda eventuais efeitos de mudanças regulatórias sobre as condições de mercado e o desempenho de empresas reguladas, precificando-os conforme prega a teoria dos mercados eficientes (Fama \& French, 1989).

Uma pesquisa preliminar indica relativa escassez de pesquisas acadêmicas acerca de efeitos de intervenções regulatórias no valor de mercado de empresas. Smith, Bradley e Jarrell (1986) encontraram evidências de perda de valor de mercado de companhias refinadoras de petróleo nos EUA em 1973, como resultado de uma mudança da política regulatória norte-americana. Su e Fleisher (1998) demonstraram aumento na volatilidade dos preços de ações de empresas chinesas, entre 1990 e 1996, em decorrência de alterações na regulação financeira, destacando diferentes magnitudes do efeito em dois mercados distintos.

Buckland e Fraser (2001) encontraram significativo aumento do risco de mercado de empresas do setor elétrico do Reino Unido após reforma regulatória introduzida em 1992. Também para o setor elétrico do Reino Unido, Paleari e Redondi (2005) evidenciaram relação negativa entre o rigor da regulação e os retornos anormais de empresas distribuidoras de energia elétrica do Reino Unido no período de 1990-1995.

Kobialka e Rammerstorfer (2009) analisaram os efeitos de 12 episódios regulatórios relacionados ao mercado de energia da Alemanha, encontrando relação destes com retornos anormais e variações do risco sistêmico para algumas empresas.

No que tange ao Brasil, Taffarel, Silva e Clemente (2013) estudaram eventuais impactos de eventos regulatórios no preço e na volatilidade das ações de oito empresas do setor de energia brasileiro, entre 2004 e 2010. Seus resultados não indicaram influência homogênea sobre todas as empresas; contudo, em determinados casos, alguns fatos regulatórios impactaram negativamente o retorno e o risco das ações das empresas. Os artigos mencionados anteriormente trazem exemplos de situações em que mudanças regulatórias tiveram impactos sobre o valor de mercado de empresas diretamente afetadas, conforme expectativa formada a partir das teorias da regulação e das finanças. Diante disso, é formulada a primeira hipótese de pesquisa:

H1: Há associação negativa entre a implementação das disposições da MP $n^{o}$ 579/2012 (Lei $n^{o}$ 12.783/2013) e o valor das ações das empresas do setor elétrico brasileiro.

\subsection{Indicadores econômico-financeiros e mercado de capitais}

Matarazzo (2010), Lagioia (2011) e Pinheiro (2016) descrevem que a avaliação das ações, alicerçada na análise econômico-financeira, contempla um conjunto de indicadores relacionados com os dados contábeis da empresa, organizados em indicadores de liquidez, de endividamento ou de estrutura de capital e de rentabilidade. Os índices de liquidez mostram a base financeira da empresa, os de endividamento medem a composição das fontes passivas de recursos da companhia, enquanto os de rentabilidade procuram evidenciar a rentabilidade do capital investido (Iudícibus, 
2017; Lagioia, 2011; Pinheiro, 2016).

Pesquisas empíricas sobre a relevância das demonstrações financeiras na identificação de tendências e no fornecimento de informações a investidores para tomada de decisões, por meio de indicadores, vêm se desenvolvendo com o passar dos anos, a partir dos trabalhos pioneiros de Beaver (1966) e Ball e Brown (1968).

A Tabela 1 apresenta alguns estudos que investigaram a relação entre indicadores econômico-financeiros e valor ou volatilidade das ações em diversos mercados acionários.

Tabela 1

\section{Estudos envolvendo indicadores econômico-financeiros e mercado de capitais}

\begin{tabular}{|c|c|c|c|c|}
\hline Autores & $\begin{array}{l}\text { Mercado } \\
\text { acionário }\end{array}$ & Amostra & Indicadores utilizados & Variáveis significativas \\
\hline Ou \& Penman (1989) & EUA & $\begin{array}{l}\text { Companhias listadas } \\
\text { na NYSE/Amex }\end{array}$ & $\begin{array}{l}\text { LC, LS, PCT, IPL, ML, } \\
\text { ROE, ROA }\end{array}$ & $\begin{array}{l}\text { LC, LS, PCT, IPL, ML, } \\
\text { ROE, ROA }\end{array}$ \\
\hline $\begin{array}{l}\text { Holthausen \& Larcker } \\
\text { (1992) }\end{array}$ & EUA & $\begin{array}{l}\text { Companhias listadas } \\
\text { na NYSE/Amex }\end{array}$ & $\begin{array}{l}\text { LC, LS, PCT, ML, } \\
\text { ROE, ROA }\end{array}$ & $\mathrm{LC}, \mathrm{LS}, \mathrm{ML}$ \\
\hline $\begin{array}{l}\text { Johnson \& Mitton } \\
(2003)\end{array}$ & Malásia & 424 empresas & $\begin{array}{l}\text { LC, LS, CE, IE, ML, } \\
\text { ROA }\end{array}$ & IE, ML, ROA \\
\hline Hobarth (2006) & EUA & 9.854 empresas & LC, LS, IE, PCT ROA & ROA \\
\hline Hamzah (2007) & Indonésia & 135 empresas fabris & LC, PCT, ROA & $\mathrm{LC}$ \\
\hline Zeitun \& Tian (2007) & Jordânia & 167 empresas & IE, PCT, ROE, ROA & IE, PCT \\
\hline King \& Santor (2008) & Canadá & 613 empresas & IE, ROA & IE, ROA \\
\hline $\begin{array}{l}\text { Martani \& } \\
\text { Khairurizka (2009) }\end{array}$ & Indonésia & $\begin{array}{l}39 \text { empresas } \\
\text { manufatureiras }\end{array}$ & LC, PCT, ML, ROE & ML, ROE \\
\hline $\begin{array}{l}\text { Sharma \& Sharma } \\
(2009)\end{array}$ & Índia & $\begin{array}{l}889 \text { empresas } \\
\text { manufatureiras }\end{array}$ & LC, PCT, ROA & PCT, ROA \\
\hline $\begin{array}{l}\text { Alexakis, Patra \& } \\
\text { Poshakwale (2010) }\end{array}$ & Grécia & 47 empresas & $\begin{array}{l}\text { LC, IE, PCT, GAT, } \\
\text { ML, ROE, ROA }\end{array}$ & $\begin{array}{l}\text { LC, IE, PCT, GAT, ML, } \\
\text { ROE, ROA }\end{array}$ \\
\hline $\begin{array}{l}\text { Riantani, Hartaya \& } \\
\text { Hasanah (2011) }\end{array}$ & Indonésia & $\begin{array}{l}11 \text { empresas de } \\
\text { mineração }\end{array}$ & PCT, ROA & PCT, ROA \\
\hline $\begin{array}{l}\text { Taani \& Banykhaled } \\
(2011)\end{array}$ & Jordânia & $\begin{array}{l}40 \text { empresas } \\
\text { manufatureiras }\end{array}$ & LC, PCT, ML, ROE & PCT, ROE \\
\hline $\begin{array}{l}\text { Goslin, Chai \& } \\
\text { Gunasekarage (2012) }\end{array}$ & $\begin{array}{l}\text { Nova } \\
\text { Zelândia } \\
\end{array}$ & 70 empresas & LC, LS, ROE, ROA & LC, LS, ROE, ROA \\
\hline $\begin{array}{l}\text { Hatta \& Dwiyanto } \\
(2012)\end{array}$ & Indonésia & 17 empresas & LC, PCT, ML, ROA & PCT, ML \\
\hline Kaveski (2013) & Brasil & $\begin{array}{l}27 \text { empresas do } \\
\text { índice IBrX-50 }\end{array}$ & $\begin{array}{l}\text { LG, LC, LS, ML, ROE, } \\
\text { ROA }\end{array}$ & $\begin{array}{l}\text { LG, LC, LS, ML, ROE, } \\
\text { ROA }\end{array}$ \\
\hline $\begin{array}{l}\text { Petcharabul \& } \\
\text { Romprasert (2014) } \\
\end{array}$ & Tailândia & $\begin{array}{l}22 \text { empresas de } \\
\text { tecnologia }\end{array}$ & LC, PCT, ROE & ROE \\
\hline Santos (2017) & Brasil & $\begin{array}{l}20 \text { instituições } \\
\text { bancárias }\end{array}$ & ML, ROE, ROA & ROE \\
\hline $\begin{array}{l}\text { Muhammad \& Ali } \\
\text { (2018) }\end{array}$ & Paquistão & $\begin{array}{l}115 \text { empresas não } \\
\text { financeiras }\end{array}$ & LC, PCT, ROA & LC, PCT, ROA \\
\hline
\end{tabular}

Amex $=$ American Stock Exchange. NYSE = New York Stock Exchange. $\mathrm{S} \& \mathrm{P}=$ Standard \& Poor's. IBrX-50= Índice Brasil 50. LG $=$ Liquidez Geral. $\mathrm{LC}=$ Liquidez Corrente. $\mathrm{LS}=$ Liquidez Seca. $\mathrm{CE}=$ Composição do Endividamento. $\mathrm{IE}=$ Índice de Endividamento. PCT = Participação de Capital de Terceiros. IPL = Imobilização do Patrimônio Líquido. GAT = Giro do Ativo Total. ML = Margem Líquida. ROE = Retorno sobre o Patrimônio Líquido. ROA = Retorno sobre o Ativo.

Fonte: Elaborado pelos autores.

Os achados dos estudos de Ou e Penman (1989), Holthausen e Larcker (1992), Hamzah (2007), Alexakis, Patra e Poshakwale (2010), Goslin, Chai e Gunasekarage (2012), Kaveski (2013) e Santos (2017) indicaram relação positiva entre indicadores de liquidez e valor das ações no mercado de capitais.

Do mesmo modo, associação positiva entre indicadores de rentabilidade e valor de ações no mercado de capitais foi constatada nos trabalhos empíricos de Hobarth (2006), Martani e Khairurizka (2009), Sharma e Sharma (2009), Alexakis, Patra e Poshakwale (2010), Riantani, 
Hartaya e Hasanah (2011), Taani e Banykhaled (2011), Goslin, Chai e Gunasekarage (2012), Kaveski (2013), Petcharabul e Romprasert (2014), Santos (2017) e Muhammad e Ali (2018).

Já as descobertas de Johnson e Mitton (2003), Zeitun e Tian (2007), King e Santor (2008), Alexakis, Patra e Poshakwale (2010), Riantani, Hartaya e Hasanah (2011) e Hatta e Dwiyanto (2012) sugeriram associação negativa entre indicadores de endividamento e valor de ações de companhias no mercado acionário.

Diante do exposto, são apresentadas as demais hipóteses de pesquisa.

$\mathbf{H}_{2}$ : Há relação positiva entre os indicadores de liquidez e o valor das ações das empresas do setor elétrico brasileiro.

$\mathbf{H}_{3}$ : Há relação negativa entre os indicadores de endividamento (estrutura de capital) e o valor das ações das empresas do setor elétrico brasileiro.

$\mathbf{H}_{4}$ : Há relação positiva entre os indicadores de rentabilidade e o valor das ações das empresas do setor elétrico brasileiro.

\section{PROCEDIMENTOS METODOLÓGICOS}

O valor de mercado da ação se refere ao preço da última cotação de cada trimestre da ação mais negociada de cada companhia, quer ordinária ou preferencial. Os indicadores econômicofinanceiros utilizados neste estudo são aqueles mais empregados nos trabalhos levantados. Dessa forma, foram utilizados índices que representam os três grupos: liquidez (LC e LS), endividamento ou estrutura de capital (IE e PCT) e rentabilidade (ROE e ROA).

Tabela 2

\section{Indicadores econômico-financeiros utilizados na pesquisa}

\begin{tabular}{|c|c|c|c|c|}
\hline \multicolumn{2}{|c|}{ Variável } & Indicador & Descrição & Fórmula \\
\hline \multirow{2}{*}{$\frac{N}{2}$} & $\mathrm{LC}$ & Liquidez Corrente & $\begin{array}{l}\text { Retrata a relação entre o ativo circulante e o } \\
\text { passivo circulante }\end{array}$ & $L C_{t}=\frac{\mathrm{AC}_{\mathrm{t}}}{\mathrm{PC}_{\mathrm{t}}}$ \\
\hline & LS & Liquidez Seca & $\begin{array}{l}\text { Revela a capacidade de cobertura do passivo } \\
\text { de curto prazo com o ativo de curto prazo, } \\
\text { desconsiderando os estoques }\end{array}$ & $L S_{t}=\frac{\mathrm{AC}_{\mathrm{t}}-\text { Estoques }_{\mathrm{t}}}{\mathrm{PC}_{\mathrm{t}}}$ \\
\hline \multirow{2}{*}{ 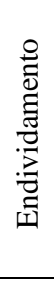 } & IE & $\begin{array}{l}\text { Índice de } \\
\text { Endividamento }\end{array}$ & $\begin{array}{l}\text { Expressa a participação do capital de } \\
\text { terceiros sobre os recursos totais (capital de } \\
\text { terceiros mais capital próprio) }\end{array}$ & $I E_{t}=\frac{\mathrm{ET}_{\mathrm{t}}}{\mathrm{ET}_{\mathrm{t}}+\mathrm{PL}_{\mathrm{t}}}$ \\
\hline & PCT & $\begin{array}{l}\text { Participação de } \\
\text { Capital de Terceiros }\end{array}$ & $\begin{array}{l}\text { Indica o nível de endividamento da empresa } \\
\text { em relação ao financiamento com recursos } \\
\text { próprios }\end{array}$ & $P C T_{t}=\frac{\mathrm{ET}_{\mathrm{t}}}{\mathrm{PL}_{\mathrm{t}}}$ \\
\hline \multirow{2}{*}{ 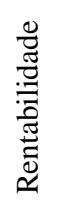 } & ROE & $\begin{array}{l}\text { Retorno sobre o } \\
\text { Patrimônio Líquido }\end{array}$ & $\begin{array}{l}\text { Mensura o retorno dos recursos aplicados } \\
\text { pelos proprietários }\end{array}$ & $R O E_{t}=\frac{\mathrm{LL}_{\mathrm{t}}}{\mathrm{PL}_{\mathrm{t}-1}}$ \\
\hline & ROA & Retorno sobre o Ativo & $\begin{array}{l}\text { Mede o retorno produzido pelo total de } \\
\text { recursos aplicados nos ativos }\end{array}$ & $R O A_{t}=\frac{\mathrm{LL}_{\mathrm{t}}}{A T M_{t}}$ \\
\hline
\end{tabular}

$\mathrm{AC}=$ Ativo Circulante. $\mathrm{PC}=$ Passivo Circulante. $\mathrm{ET}=$ Endividamento Total. $\mathrm{PL}=$ Patrimônio Líquido. LL $=$ Lucro Líquido. $\mathrm{AT}=$ Ativo Total. $\mathrm{ATM}=$ Ativo Total Médio $\left(\left(\mathrm{AT}_{\mathrm{t}}+\mathrm{AT}_{\mathrm{t}-1}\right) / 2\right) \cdot \mathrm{t}=$ indicador do período atual. $\mathrm{t}-1=$ indicador do período anterior.

Fonte: Lagioia (2011), Iudícibus (2017), Martins, Diniz \& Miranda (2018).

Foi utilizado modelo econométrico de regressão múltipla para dados em painel para análise dos efeitos da regulação econômica implementada pela MP n ${ }^{\circ}$ 579/2012 sobre o valor de mercado das ações das empresas do setor elétrico brasileiro, bem como o nível de relação entre indicadores econômico-financeiros e o valor das ações no mercado de capitais, no período entre 2009 e 2016. 
Gujarati e Porter (2011) frisam que dados em painel podem ser explorados utilizando-se de três técnicas de estimação. O modelo Mínimos Quadrados Ordinários (MQO) para dados empilhados ou Pooled Ordinary Least Squares (POLS) simplesmente empilha as observações em uma grande regressão, desprezando as características dos dados quanto à natureza de corte transversal e de série temporal. O Modelo de Efeitos Fixos (MEF) pressupõe que o intercepto da regressão pode diferir entre os indivíduos, mas não varia com o tempo (cada unidade de corte transversal tem um intercepto próprio com valor fixo). O Modelo de Efeitos Aleatórios (MEA) ou Modelo de Componentes de Erros (MCE) pressupõe que o intercepto seja uma variável aleatória comum entre os indivíduos (valor médio de todos os interceptos) e as diferenças individuais de cada sujeito se refletem no termo de erro.

Wooldridge (2016) entende que as aplicações de inferência estatística com dados em painel devem distinguir as diferenças entre esses modelos, em virtude da suposta existência de informação suficiente nos dados para produzir estimativas precisas dos coeficientes. Portanto, a averiguação sobre a modelagem em painel que melhor se adequa a determinado conjunto de dados foi realizada por meio dos testes da Tabela 3.

Tabela 3

Testes para seleção do modelo para dados em painel

\begin{tabular}{l|c|c|c}
\hline & Teste F de Chow & Teste LM de Breusch-Pagan & Teste de Hausman \\
\hline Seleção do modelo & POLS versus MEF & POLS versus MEA & MEF versus MEA \\
\hline \multirow{2}{*}{ Critério de seleção } & $\mathrm{H}_{0}:$ método POLS & $\mathrm{H}_{0}:$ método POLS & $\mathrm{H}_{0}:$ método MEA \\
& $\mathrm{H}_{1}:$ método MEF & $\mathrm{H}_{1}:$ método MEA & $\mathrm{H}_{1}:$ método MEF \\
\hline
\end{tabular}

Nota. $\mathrm{H}_{0}$ : Hipótese nula. $\mathrm{H}_{1}$ : Hipótese alternativa.

Fonte: Fávero \& Belfiore (2017), Gujarati \& Porter (2011) e Wooldridge (2016).

O modelo de regressão múltipla para dados em painel utilizado neste estudo é:

$$
\begin{aligned}
V M A_{i, t}= & \alpha+\beta_{1} L C_{i, t}+\beta_{2} L S_{i, t}+\beta_{3} I E_{i, t}+\beta_{4} P C T_{i, t}+\beta_{5} R O E_{i, t}+\beta_{6} R O A_{i, t}+ \\
& +\beta_{7} M P_{i, t}+\mu_{i, t}
\end{aligned}
$$

em que, $V M A_{i, t}$ representa o valor de mercado da ação da empresa $i$ no trimestre $t ; i$ representa as empresas $(i=1 \ldots 29) ; t$ corresponde aos trimestres $(t=1 \ldots 32) ; \alpha$ é o intercepto ou constante da regressão; $\beta$ são os coeficientes angulares de cada variável; $L C_{i, t}$ é o índice de Liquidez Corrente da empresa $i$ no trimestre $t ; L S_{i, t}$ é o índice de Liquidez Seca da empresa $i$ no trimestre $t$; $I E_{i, t}$ é o Índice de Endividamento da empresa $i$ no trimestre $t ; P C T_{i, t}$ é o indicador de Participação de Capital de Terceiros da empresa $i$ no trimestre $t ; R O E_{i, t}$ é o indicador de Retorno sobre o Patrimônio Líquido da empresa $i$ no trimestre $t$; $R O A_{i, t}$ é o indicador de Retorno sobre o Ativo da empresa $i$ no trimestre $t ; M P_{i, t}$ é a variável qualitativa (binária) que capta o efeito da Medida Provisória ${ }^{\circ} 579 / 2012$, Dummy 0 para o período pré-MP (primeiro trimestre de 2009 ao terceiro trimestre de 2012) e Dummy 1 para o pós-MP (quarto trimestre de 2012 ao quarto trimestre de 2016); e $\mu_{i, t}$ corresponde ao erro idiossincrático ou termo de erro.

A descrição das variáveis explicativas utilizadas nesta pesquisa e os respectivos sinais esperados estão descritos resumidamente na Tabela 4. 
Tabela 4

Variáveis explicativas da pesquisa

\begin{tabular}{|c|c|c|c|c|}
\hline \multicolumn{2}{|c|}{ Variável } & Indicador & Embasamento empírico & $\begin{array}{l}\text { Sinal } \\
\text { esperado }\end{array}$ \\
\hline \multirow[t]{2}{*}{$\frac{N}{\frac{N}{\sigma}}$} & $\mathrm{LC}$ & Liquidez Corrente & $\begin{array}{l}\text { Ou e Penman (1989); Hamzah (2007); Alexakis, } \\
\text { Patra e Poshakwale (2010); Goslin, Chai e } \\
\text { Gunasekarage (2012); Kaveski (2013); Santos } \\
\text { (2017). }\end{array}$ & Positivo \\
\hline & $\mathrm{LS}$ & Liquidez Seca & $\begin{array}{l}\text { Ou e Penman (1989); Holthausen e Larcker (1992) } \\
\text { Kaveski (2013); Santos (2017). }\end{array}$ & Positivo \\
\hline \multirow{2}{*}{ 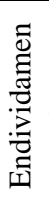 } & IE & Índice de Endividamento & $\begin{array}{l}\text { Johnson e Mitton (2003); Zeitun e Tian (2007); King } \\
\text { e Santor (2008). }\end{array}$ & Negativo \\
\hline & PCT & Participação de Capital de Terceiros & $\begin{array}{l}\text { Zeitun e Tian (2007); Alexakis, Patra e Poshakwale } \\
\text { (2010); Riantani, Hartaya e Hasanah (2011); Hatta e } \\
\text { Dwiyanto (2012). }\end{array}$ & Negativo \\
\hline \multirow{2}{*}{ 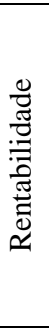 } & ROE & Retorno sobre o Patrimônio Líquido & $\begin{array}{l}\text { Martani e Khairurizka (2009); Alexakis, Patra e } \\
\text { Poshakwale (2010); Taani e Banykhaled (2011); } \\
\text { Goslin, Chai e Gunasekarage (2012); Kaveski (2013); } \\
\text { Petcharabul e Romprasert (2014); Santos (2017). }\end{array}$ & Positivo \\
\hline & ROA & Retorno sobre o Ativo & $\begin{array}{l}\text { Hobarth (2006); Sharma e Sharma (2009); Alexakis, } \\
\text { Patra e Poshakwale (2010); Riantani, Hartaya e } \\
\text { Hasanah (2011); Kaveski (2013); Muhammad e Ali } \\
\text { (2018). }\end{array}$ & Positivo \\
\hline 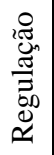 & MP & $\begin{array}{l}\text { Medida Provisória 579/2012 } \\
\text { (variável dummy) }\end{array}$ & $\begin{array}{l}\text { Smith, Bradley e Jarrell (1986); Paleari e Redondi } \\
\text { (2005); Cerqueira (2013); Prado e Silva (2013); } \\
\text { Taffarel, Silva e Clemente (2013). }\end{array}$ & Negativo \\
\hline
\end{tabular}

Fonte: Elaborado pelos autores.

A amostra de pesquisa compreende as 29 companhias titulares de concessão/permissão de geração, transmissão e/ou distribuição de energia elétrica que apresentaram ações negociadas e os indicadores econômico-financeiros selecionados, no período de 2009 a 2016. A Tabela 5 especifica a amostra de pesquisa.

Tabela 5

\section{Empresas componentes da amostra}

\begin{tabular}{|c|c|c|c|}
\hline Empresa & Ação & Empresa & Ação \\
\hline 1. AES Tietê Energia S.A. & TIET3 & $\begin{array}{l}\text { 16. Companhia Estadual de Geração e } \\
\text { Transmissão de Energia Elétrica }\end{array}$ & EEEL3 \\
\hline 2. Afluente Transmissora de Energia S.A. & AFLU3 & 17. Companhia Paranaense de Energia & CPLE3 \\
\hline 3. Ampla Energia e Serviços S.A. & CBEE3 & 18. CPFL Energia S.A. & CPFE3 \\
\hline 4. Centrais Elétricas Brasileiras S.A. & ELET3 & $\begin{array}{l}\text { 19. Companhia de Transmissão de } \\
\text { Energia Elétrica Paulista }\end{array}$ & TRPL3 \\
\hline 5. Centrais Elétricas de Santa Catarina S.A. & CLSC4 & 20. EDP Energia do Brasil S.A. & ENBR3 \\
\hline 6. Centrais Elétricas do Pará S.A. & CELP5 & 21. Elektro Redes S.A. & EKTR4 \\
\hline 7. Companhia Energética de São Paulo & CESP3 & $\begin{array}{l}\text { 22. Eletropaulo Metropolitana Eletric. de } \\
\text { São Paulo S.A. }\end{array}$ & ELPL3 \\
\hline 8. Companhia de Eletricidade do Estado da Bahia & CEEB3 & $\begin{array}{l}\text { 23. Empresa Metropolitana de Águas e } \\
\text { Energia S.A. }\end{array}$ & EMAE4 \\
\hline 9. Companhia Energética de Brasília & CEBR3 & 24. Energisa S.A. & ENGI3 \\
\hline 10. Companhia Energética de Minas Gerais & CMIG3 & 25. Eneva S.A. & ENEV3 \\
\hline 11. Companhia Energética de Pernambuco & CEPE5 & 26. Engie Brasil Energia S.A. & EGIE3 \\
\hline 12. Companhia Energética do Ceará & COCE3 & 27. Equatorial Energia S.A. & EQTL3 \\
\hline 13. Companhia Energética do Maranhão & ENMA3 & 28. Rio Paranapanema Energia S.A. & GEPA4 \\
\hline 14. Companhia Energética do Rio G. do Norte & CSRN3 & 29. Companhia Estadual de Distribuição & CEED3 \\
\hline 15. Light S.A. & LIGT3 & de Energia Elétrica & \\
\hline
\end{tabular}

Fonte: B3 S.A. (2017). 
O período de análise se refere ao intervalo entre o primeiro trimestre de 2009 e o quarto trimestre de 2016, compreendendo as informações individuais e trimestrais das companhias da amostra. A coleta dos valores das ações das empresas e dos indicadores econômico-financeiros foi realizada na página eletrônica da B3 e na base de dados da Economática.

\section{RESULTADOS}

Nesta seção são apresentados os resultados auferidos no estudo, destacando os testes de validação do modelo econométrico, a análise dos resultados das estimações da regressão, assim como a discussão dos resultados diante dos pressupostos teóricos e das pesquisas empíricas.

\subsection{Testes e análise dos resultados das estimações}

Os achados de risco de multicolinearidade podem ser observados por intermédio da matriz de correlação de Pearson, dispostos na Tabela 6.

Tabela 6

Matriz de Correlação de Pearson

\begin{tabular}{r|rrrrrrr}
\hline & LC & LS & IE & PCT & ROE & ROA & MP \\
\hline LC & 1,0000 & & & & & & \\
LS & $1,0000^{*}$ & 1,0000 & & & & & \\
IE & $-0,3355$ & $-0,3356$ & 1,0000 & & & & \\
PCT & $-0,0211$ & $-0,0211$ & 0,0845 & 1,0000 & & & \\
ROE & 0,0107 & 0,0107 & $-0,0154$ & $-0,0010$ & 1,0000 & & \\
ROA & $-0,0370$ & $-0,0369$ & $-0,1877$ & $-0,0332$ & $-0,2944$ & 1,0000 & \\
MP & $-0,0703$ & $-0,0703$ & 0,1007 & $-0,0076$ & 0,0332 & $-0,1418$ & 1,0000 \\
\hline
\end{tabular}

Nota. * Correlação significativa $>0,8$.

Fonte: Elaborado pelos autores.

Conforme dados dessa matriz, evidencia-se correlação linear significativa entre Liquidez Corrente (LC) e Liquidez Seca (LS). Tendo em vista que o índice de Liquidez Corrente é mais empregado em trabalhos empíricos que pesquisam índices de liquidez, optou-se por excluir a variável Liquidez Seca do modelo de estimação.

A Tabela 7 mostra o resultado da aplicação do teste de multicolinearidade VIF (Variance Inflation Factor) sem a variável LS, revelando a inexistência de multicolinearidade dos regressores.

Tabela 7

Teste estatística VIF

\begin{tabular}{rrr}
\hline Variável & VIF & $1 / \mathrm{VIF}$ \\
\hline IE & 1,20 & 0,834585 \\
ROA & 1,17 & 0,851378 \\
LC & 1,14 & 0,873844 \\
ROE & 1,10 & 0,907393 \\
MP & 1,03 & 0,971096 \\
PCT & 1,01 & 0,971096 \\
\hline Mean VIF & 1,11 & \\
\hline
\end{tabular}

Nota. VIF > 10 = Correlação entre variáveis (Gujarati \& Porter, 2011, Wooldridge, 2016).

Fonte: Elaborado pelos autores.

Após diagnóstico de multicolinearidade, o modelo de estimação para explicar o valor de mercado das ações das empresas é dado pela Equação 1, excluindo a variável Liquidez Seca.

Examinou-se, ainda, a modelagem que melhor se ajusta aos dados da pesquisa, por meio da comparação entre os estimadores do modelo POLS, Modelo de Efeitos Fixos (MEF) ou Método de Efeitos Aleatórios (MEA). Os resultados dos testes estão descritos na Tabela 8. 
Tabela 8

Testes de seleção do modelo para dados em painel

\begin{tabular}{l|c|c|c}
\hline & Teste F de Chow & Teste LM de Breusch-Pagan & Teste de Hausman \\
\hline Seleção do modelo & POLS versus MEF & POLS versus MEA & MEF versus MEA \\
\hline \multirow{2}{*}{ Critério de seleção } & $\mathrm{H}_{0}:$ método POLS & $\mathrm{H}_{0}:$ método POLS & $\mathrm{H}_{0}:$ método MEA \\
& $\mathrm{H}_{1}:$ método MEF & $\mathrm{H}_{1}:$ método MEA & $\mathrm{H}_{1}:$ método MEF \\
\hline \multirow{2}{*}{ Resultado do teste } & $\mathrm{F}(28,893)=27,91$ & chibar2 $(01)=2869,28$ & chi2 $(6)=1,87$ \\
& Prob $>\mathrm{F}=0,0000$ & Prob $>$ chibar2 $=0,0000$ & Prob chi $2=0,7600$ \\
\hline Análise do teste & Rejeita $\mathrm{H}_{0}$ & Rejeita $\mathrm{H}_{0}$ & Não rejeita $\mathrm{H}_{0}$ \\
\hline
\end{tabular}

Fonte: Dados elaborados pelos autores.

Conforme resultados dos testes, o Modelo de Efeitos Aleatórios (MEA) ou Modelo de Componentes de Erros (MCE) é mais apropriado para se investigar o comportamento do valor das ações das empresas desta pesquisa.

Os testes de Breusch-Pagan e de Wooldridge para dados em painel indicaram a presença de heterocedasticidade e de autocorrelação dos erros, conforme dados da Tabela 9.

Tabela 9

Resultados dos testes para heterocedasticidade e autocorrelação

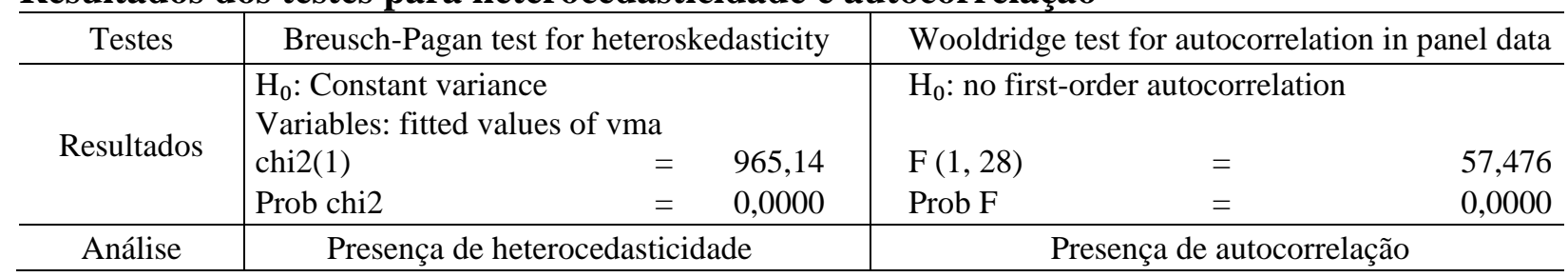

Fonte: Elaborado pelos autores.

Nos casos de ausência de homocedasticidade dos erros (heterocedasticidade), Gujarati e Porter (2011) e Wooldridge (2016) destacam, entre as várias medidas corretivas, a utilização de estimadores pelo método de Mínimos Quadrados Generalizados (MQG) ou Mínimos Quadrados Ponderados (MQP). Nesse sentido, dado que os testes apontaram o Método de Efeitos Aleatórios (MEA) a ser utilizado, ter-se-á atendida a correção da heterocedasticidade dos resíduos, pois o MEA estima parâmetros por MQG.

Dentre os muitos procedimentos para tratar a correlação serial dos resíduos, Gujarati e Porter (2011), Wooldridge (2016) e Fávero e Belfiore (2017) sugerem o procedimento de efeitos autorregressivos de primeira ordem $\mathrm{AR}(1)$ dos termos de erro.

Wooldridge (2016) frisa que o modelo de regressão, na presença de heterocedasticidade e autocorrelação, pode ser estimado por meio de um procedimento combinado de autorregressivo AR(1) e Mínimos Quadrados Ponderados (MQP), corrigindo os dois problemas. Desse modo, os resultados da regressão pelo MEA, estimado por mínimos quadrados generalizados com o procedimento AR(1) dos resíduos, estão dispostos na Tabela 10.

Tabela 10

Resultados da estimação pelo modelo MEA com AR(1)

\begin{tabular}{|c|c|c|c|c|c|c|c|c|}
\hline \multicolumn{5}{|c|}{ RE GLS regression with AR(1) disturbances } & & Number of obs & $=$ & 928 \\
\hline \multicolumn{6}{|c|}{ Group variable: id } & Number of grups & $=$ & 29 \\
\hline \multirow[t]{4}{*}{ R-sq: } & within & $=$ & 0,1169 & & & Obs per group: $\min$ & $=$ & 32 \\
\hline & between & $=$ & 0,0428 & & & avg & $=$ & 32,0 \\
\hline & overall & $=$ & 0,0776 & & & $\max$ & $=$ & 32 \\
\hline & & & & & & Wald chi2(7) & $=$ & 34,94 \\
\hline \multicolumn{2}{|c|}{$\operatorname{corr}\left(\mathrm{u} \_\mathrm{i}, \mathrm{Xb}\right)$} & $=$ & 0 (assumed) & & & Prob F & $=$ & 0,0000 \\
\hline vma & Coef. & & Std. Err. & $\mathrm{Z}$ & $\mathrm{P}>|\mathrm{z}|$ & \multicolumn{2}{|c|}{ [95\% Conf. Interval] } & Sig. \\
\hline lc & 0,319338 & & 0,083813 & 3,81 & 0,000 & 0,155067 & 608 & $*$ \\
\hline ie & $-0,099474$ & & 0,050330 & $-1,98$ & 0,048 & $-0,198119$ & $-0,000829$ & $* *$ \\
\hline
\end{tabular}




\begin{tabular}{r|rrrrrr} 
pct & $-0,000015$ & 0,000144 & $-0,10$ & 0,917 & $-0,000297$ & 0,000267 \\
roe & 0,000814 & 0,001273 & 0,64 & 0,523 & $-0,001682$ & 0,003310 \\
roa & 0,027682 & 0,073551 & 0,38 & 0,707 & $-0,116475$ & 0,171839 \\
mp & $-3,946458$ & 1,285478 & $-3,07$ & 0,002 & $-6,465948$ & $-1,426968$ \\
cons & 26,504340 & 3,042232 & 8,71 & 0,000 & 20,54168 & 32,46701 \\
Nota. * e ** indicam que as variáveis são estatisticamente significantes ao nível de 1\% e 5\%, respectivamente. \\
Fonte: Elaborado pelos autores.
\end{tabular}

Os resultados do modelo de estimação para o valor de mercado das ações das companhias do setor elétrico brasileiro indicam que LC e MP são estatisticamente significativas ao nível de $1 \%$ e IE é estatisticamente significante a $5 \%$. Os achados apontam que as variáveis quantitativas de Liquidez Corrente e de Índice de Endividamento e a variável qualitativa que mede o impacto da MP $n^{\circ} 579 / 2012$ possuem relação estatisticamente significativa com o valor das ações das empresas concessionárias de energia elétrica.

Após a realização dos testes e análise das estimações, verificou-se que o modelo econométrico é estatisticamente significativo (Prob $\mathrm{F}$ menor que $5 \%$ ), no qual $7,76 \%$ do comportamento da variação geral do valor de mercado das ações é devido à variação conjunta das variáveis explicativas, alcançando 11,69\% de poder de explicação dentro dos painéis.

\subsection{Discussão dos resultados}

Com base nas hipóteses propostas, a Tabela 11 apresenta os resultados.

Tabela 11

\section{Resumo dos resultados das hipóteses de pesquisa}

\begin{tabular}{|c|c|}
\hline Hipóteses & Resultado \\
\hline $\begin{array}{l}\text { H}_{1} \text { : Há associação negativa entre a implementação das disposições da MP n }{ }^{\circ} \text { 579/2012 (Lei no } \\
\text { 12.783/2013) e o valor das ações das empresas do setor elétrico brasileiro. }\end{array}$ & Não rejeita \\
\hline $\begin{array}{l}\mathrm{H}_{2} \text { : Há relação positiva entre indicador(es) de liquidez e o valor das ações das empresas do setor } \\
\text { elétrico brasileiro. }\end{array}$ & Não rejeita \\
\hline $\begin{array}{l}\mathrm{H}_{3} \text { : Há relação negativa entre indicador(es) de endividamento (estrutura de capital) e o valor das } \\
\text { ações das empresas do setor elétrico brasileiro. }\end{array}$ & Não rejeita \\
\hline $\begin{array}{l}\mathrm{H}_{4} \text { : Há relação positiva entre indicador(es) de rentabilidade e o valor das ações das empresas do setor } \\
\text { elétrico brasileiro. }\end{array}$ & Rejeita \\
\hline
\end{tabular}

Fonte: Elaborado pelos autores.

Conforme resultados apurados, constatou-se associação negativa entre as disposições implementadas pela MP n 579/2012 (Lei n ${ }^{\circ} 12.783 / 2013$ ) e o valor das ações das empresas do setor elétrico brasileiro no mercado de capitais, ou seja, a entrada em vigor deste preceito regulatório acarretou decréscimos de valor de mercado das ações das companhias concessionárias de energia elétrica. Assim, não se pode rejeitar a hipótese 1, pois os resultados indicaram evidências estatisticamente significativas da associação esperada.

Os achados da associação negativa entre a MP n 579/2012 e o valor de mercado das ações das firmas se assemelham às descobertas de Smith, Bradley e Jarrell (1986), Paleari e Redondi (2005), Cerqueira (2013), Prado e Silva (2013) e Taffarel, Silva e Clemente (2013).

Pressupostos teóricos sugerem que os efeitos negativos no mercado de capitais em relação aos segmentos regulados podem estar associados a impactos de eventos regulatórios, com reflexos no valor de mercado das firmas (Paleari \& Redondi, 2005). Os resultados desta pesquisa apontaram que efeitos adversos da MP n ${ }^{\circ}$ 579/2012 repercutiram nas empresas titulares de concessão de geração, transmissão e/ou distribuição de energia elétrica no país, no tocante à perda de valor de mercado das companhias.

Ademais, segundo Buchanan e Tullock (1962), decisões políticas e econômicas de entes governamentais estão sujeitas a diferentes poderes exercidos por vários agentes dentro do mesmo sistema político, interferindo na implementação dessas decisões, cujos impactos podem ser os mais variados possíveis. Ao propor essa intervenção no mercado de energia elétrica com o intuito de viabilizar a redução da tarifa para o consumidor, a decisão estatal, por meio da MP nº 579/2012, 
provocou efeitos danosos às empresas no setor.

No que concerne à segunda hipótese, na qual se analisou a relação positiva entre os indicadores de liquidez e o valor das ações das empresas do setor elétrico brasileiro, as constatações ratificaram a hipótese, uma vez que Liquidez Corrente (LC) apresentou relação estatisticamente significativa e positiva com o valor de mercado das ações. Os incrementos no índice LC indicaram correlação com elevações de valor das ações das empresas analisadas no mercado de capitais. Tais resultados corroboram os achados de Ou e Penman (1989), Hamzah (2007), Alexakis, Patra e Poshakwale (2010), Goslin, Chai e Gunasekarage (2012), Kaveski (2013) e Santos (2017), que apontaram relação positiva entre Liquidez Corrente e valor de ações no mercado acionário.

Capelletto e Corrar (2008) e Gitman (2010) salientam que as sociedades por ações podem usar indicadores financeiros, como os de liquidez, para analisar e monitorar seu desempenho, posto que repercussões sistêmicas atreladas a dificuldades de liquidez provocam queda de preços e desvalorização de ativos no mercado de ações. A solidez financeira pode prever retornos anormais e demandas futuras de investidores, assim, aumentos nos indicadores de liquidez são bons preditores de valorização de ações das empresas (Choi \& Sias, 2012), justificando a presença dessa relação significativa.

A terceira hipótese buscou averiguar a relação negativa entre os indicadores de endividamento ou de estrutura de capital e o valor das ações das companhias. A hipótese não foi rejeitada em virtude da existência da relação negativa e significativa entre a variável Índice de Endividamento (IE) e o valor das ações das companhias prestadoras de serviços de energia elétrica no período analisado. As evidências observadas neste estudo coincidem com as pesquisas de Johnson e Mitton (2003), Zeitun e Tian (2007) e King e Santor (2008).

Um crescimento do índice de endividamento pode denotar, segundo Assaf (2014), redução de rentabilidade e, por conseguinte, reflexos no mercado. Empresas com elevados índices de alavancagem tendem a captar recursos de terceiros quando suas avaliações de mercado estão em baixa (Baker \& Wurgler, 2002).

No que tange à quarta hipótese de pesquisa, que examinou a suposta relação positiva entre os indicadores de rentabilidade e o valor das ações das empresas do setor elétrico brasileiro, esta foi rejeitada. Nenhum indicador de rentabilidade apresentou qualquer conexão com o valor de mercado das empresas pertencentes à amostra, refutando assim a hipótese 4. As conclusões se contrapõem aos achados de Hobarth (2006), Martani e Khairurizka (2009), Sharma e Sharma (2009), Alexakis, Patra e Poshakwale (2010), Riantani, Hartaya e Hasanah (2011), Taani e Banykhaled (2011), Goslin, Chai e Gunasekarage (2012), Kaveski (2013), Petcharabul e Romprasert (2014), Santos (2017) e Muhammad e Ali (2018), que observaram resultados estatisticamente significativos nessa relação. Este resultado pode estar relacionado com a política tarifária das companhias do segmento de energia elétrica brasileiro, seja por regime de tarifas reguladas ou por receita anual permitida, que restringe a rentabilidade das empresas, com reflexos nas receitas e, consequentemente, na geração de lucro. Por conseguinte, análises e percepções do mercado de ações seriam pouco sensíveis às alterações dos indicadores de rentabilidade das empresas desse segmento da economia.

\section{CONCLUSÕES}

A Medida Provisória n ${ }^{\circ}$ 579/2012 (Lei n ${ }^{\circ} 12.783 / 2013$ ), instituída pelo governo federal em 2012, alterou regras da legislação regulatória do segmento de energia elétrica brasileiro. A implementação da medida apontou para uma ampla repercussão no mercado de capitais, no que se refere à redução do valor das ações das empresas de energia listadas na B3, além da desconfiança de investidores locais e estrangeiros. Outrossim, especialistas levantaram aspectos vivenciados pelo setor e relacionados com a edição dessa medida regulatória, principalmente quanto ao 
equilíbrio econômico-financeiro das empresas.

Nesse contexto, a presente pesquisa teve como objetivo analisar o relacionamento entre o desempenho econômico-financeiro e o valor das ações das empresas do setor elétrico brasileiro no mercado de capitais, correlacionado com o impacto da regulação econômica disposta pela MP $n^{\circ} 579 / 2012$.

De início, os resultados indicaram que a queda no valor das ações das empresas do setor elétrico brasileiro está associada à entrada em vigor das disposições da MP no 579/2012, confirmando a premissa de efeitos maléficos dessa intervenção regulatória sobre o valor de mercado das empresas.

Esses resultados se coadunam com os argumentos de que o livre mercado seria a representação ideal do interesse público, pois, seja na política ou no mercado, indivíduos influenciados por grupos de interesse se comportam motivados em maximizar o interesse próprio em detrimento de alguma dimensão relacionada ao interesse público, de modo que a intervenção regulatória somente deveria ocorrer quando fosse minimamente necessária.

Ademais, os achados demonstraram que os índices de liquidez de curto prazo, precursores de complicações financeiras e de indícios de problemas de caixa e insolvência, apresentam-se como bons preditores para análises de mercado e demandas de investidores, mesmo em empresas de setores regulados, além de servirem de referência para expectativas de rendimentos futuros no mercado acionário.

Quanto ao índice de endividamento, um crescimento desse indicador pode denotar redução de rentabilidade e, por conseguinte, reflexos negativos no mercado, pois uma maior participação de recursos de terceiros irá aumentar as despesas financeiras, deteriorando a rentabilidade das empresas. Para tanto, é importante que se analise o custo do endividamento em relação ao retorno produzido pelos ativos financiados por esses recursos, de forma que a participação do capital de terceiros não determine uma situação de liquidez insustentável.

No tocante à rentabilidade das empresas, as restrições regulatórias sobre as receitas das companhias de energia elétrica, seja por tarifas reguladas ou receita anual permitida, com reflexos na geração de lucro, indicam estarem associadas à insensibilidade das expectativas do mercado de capitais diante das oscilações dos índices de rentabilidade de empresas desse setor.

A pesquisa, com efeito, contribui ao destacar a necessidade de se considerar os eventuais impactos sobre o mercado de alterações regulatórias, sugerindo o emprego de uma análise multivariada ao considerar os efeitos simultâneos de um evento e de variáveis econômicofinanceiras sobre o valor de mercado de empresas de capital aberto. Em particular, os resultados e conclusões obtidos contribuem com o melhor entendimento do setor elétrico brasileiro, aspecto importante para o aprimoramento da regulação do setor, ao comprovar empiricamente que o mercado reage ao conteúdo informacional de intervenções regulatórias juntamente com o de outras variáveis típicas de mercado. Ademais, espera-se que este conhecimento contribua para aprimorar o processo de análise de impacto regulatório realizado antes da edição de novas normas por parte de entes estatais.

Quanto às limitações de pesquisa, estas decorrem da não inclusão de variáveis macroeconômicas e/ou variáveis de controle que similarmente poderiam ter relação com o mercado de ações. Além do mais, erros de mensuração das variáveis podem levar a distorções e tornar inconsistente a estimação dos parâmetros do modelo de regressão.

Por fim, como sugestão para novos trabalhos, propõe-se a extensão do modelo de pesquisa a eventos regulatórios de outros setores da economia brasileira. Sugere-se, ainda, alterar o conjunto de indicadores econômico-financeiros e/ou incluir variáveis macroeconômicas e de controle ao modelo de estimação. 


\section{REFERÊNCIAS}

Alexakis, C., Patra, T., \& Poshakwale, S. (2010). Predictability of stock returns using financial statement information: evidence on semi-strong efficiency of emerging Greek stock market. Applied Financial Economics, 20(16), 1321-1326.

Assaf, A., Neto (2014). Finanças corporativas e valor (7a ed.). São Paulo: Atlas.

Baker, M., \& Wurgler, J. (2002). Market timing and capital structure. The Journal of Finance, 57(1), 1-32.

Baldwin, R., Cave, M., \& Lodge, M. (2012). Understanding regulation (2a ed.). New York: Oxford University Press.

Ball, R., \& Brown, P. (1968) An empirical evaluation of accounting income numbers. Journal of Accounting, 159-178.

Batista, H. G., \& Lima, N., Neto (2014). Ingerência do governo na Eletrobras ajuda a causar rombo de $R \$ 13$ bi desde 2012. Recuperado em 10 janeiro, 2018 de https://oglobo.globo.com/economia/ingerencia-do-governo-na-eletrobras-ajuda-causarrombo-de-13-bi-desde-2012-12380463

Beaver, W. H. (1966). Financial ratios as predictors of failure. Journal of Accounting Research, 4 , 71-111.

Becker, G. S. (1983). A theory of competition among pressure groups for political influence. The Quarterly Journal of Economics, 98(3), 371-400.

Borges, A. (2001). Democracia vs. eficiência: a teoria da escolha pública. Lua Nova, 53, 159-179.

Buchanan, J. M., \& Tullock, G. (1962). The calculus of consent. Ann Arbor: University of Michigan Press.

Buckland, R., \& Fraser, P. (2001). Political and Regulatory Risk: Beta Sensitivity in U.K. Electricity Distribution. Journal of Regulatory Economics, 19(1), 5-25.

Capelletto, L. R., \& Corrar, L. J. (2008). Índices de risco sistêmico para o setor bancário. Revista Contabilidade \& Finanças, 19(47), 6-18.

Cerqueira, M. N. F. (2013). Normas regulatórias brasileiras para incentivar o investimento privado no setor de geração de energia elétrica na última década: do modelo à realidade. Dissertação de Mestrado Profissional em Regulação e Gestão de Negócios, Universidade de Brasília, Brasília, DF, Brasil.

Fama, E. F., \& French, K. R. (1989). Business conditions and expected returns on stock markets and bonds. Journal of Financial Economics, 25(1), 23-49.

Fávero, L. P., \& Belfiore, P. (2017). Manual de análise de dados (1a ed.). Rio de Janeiro: Elsevier. 
Freire, F. S., Costa, G. P. C. L., Gartner, I. R., \& Clemente, A. (2013). As escolhas públicas orçamentárias federais no PPA 2008-2011: uma análise da perspectiva do modelo principalagente. Revista de Administração Pública, 47(5), 1089-1116.

Gitman, L. J. (2010). Princípios de administração financeira (12a ed.). São Paulo: Pearson Prentice Hall.

Godoi, M. (2013). MP 579: um ano do 11/09 do setor elétrico. Recuperado em 15 dezembro, 2017 de http://www.epe.gov.br/imprensa/Clipping/20130906_a.pdf

Goslin, J., Chai, D., \& Gunasekarage, A. (2012). The usefulness of financial statement information in predicting stock returns: New Zealand evidence. Australasian Accounting, Business and Finance Journal, 6(2), 51-70.

Gujarati, D. N., \& Porter, D. C. (2011). Econometria básica (5a ed.). Porto Alegre: McGrawHill.

Hamzah, A. (2007). Analisis rasio likuiditas, profitabilitas, aktivitas, solvabilitas dan investment opportunity set dalam tahapan siklus kehidupan perusahaan manufaktur yang terdaftar di bursa efek jakarta (bej) tahun 2001 - 2005. Jurnal Ilmiah Akuntansi dan Bisnis, 2(2), 1-22.

Hatta, A. J., \& Dwiyanto, B. S. (2012). The company fundamental factors and systematic risk in increasing stock price. Journal of Economics, Business, and Accountancy Ventura, 15(2), 245256.

Hobarth, L. L. (2006). Modeling the relationship between financial indicators and company performance. An empirical study for US-listed companies. Doctoral thesis, WU Vienna University of Economics and Business, Vienna, Austria.

Holthausen, R. W., \& Larcker, D. F. (1992). The prediction of stock returns using financial statement information. Journal of Accounting and Economics, 15(2-3), 373-411.

Iudícibus, S. de (2017). Análise de balanços (11a ed. rev. e atual.). São Paulo: Atlas.

Johnson, S., \& Mitton, T. (2003). Cronyism and capital controls: evidence from Malaysia. Journal of Financial Economics, 67(2), 351-382.

Kaveski, I. D. S. (2013). Grau de relacionamento entre indicadores de mercado de capitais, os indicadores econômicos-financeiro e o retorno da ação nas empresas brasileiras. Dissertação de Mestrado em Ciências Contábeis, Universidade Regional de Blumenau, Blumenau, SC, Brasil.

King, M. R., \& Santor, E. (2008). Family values: Ownership structure, performance and capital structure of Canadian firms. Journal of Banking \& Finance, 32(11), 2423-2432.

Kobialka, M., \& Rammerstorfer, M. (2009). Regulatory risk and market reactions - empirical evidence from Germany. Zeitschrift für Energiewirtschaft, 33(3), 221-227.

Laffont, J. J. (1994). The new economics of regulation ten years after. Econometrica, 62(3), 507537. 
Laffont, J. J., \& Tirole, J. (1990).The politics of government decision making: regulatory institutions. Journal of Law, Economics, \& Organization, 6(1), 1-31.

Lagioia, U. C. T. (2011). Fundamentos do mercado de capitais (3a ed.). São Paulo: Atlas.

Martani, M. D., \& Khairurizka, R. (2009). The effect of financial ratios, firm size, and cash flow from operating activities in the interim report to the stock return. Chinese Business Review, $8(6), 44-55$.

Martins, E., Diniz, J. A., Miranda, G. J. (2018) Análise avançada das demonstrações contábeis: uma abordagem crítica (2a ed.). São Paulo: Atlas.

Matarazzo, D. C. (2010). Análise financeira de balanços (7a ed.). São Paulo: Atlas.

Ministério de Minas e Energia - MME, Ministério da Fazenda-MF, \& Advocacia-Geral da União - AGU. (2012). EM Interministerial no 37/MME/MF/AGU, Brasília.

Mueller, B. (2001). Regulação, informação e política: uma resenha da teoria política positiva da regulação. Revista Brasileira de Economia de Empresas, 1(1), 1-21.

Muhammad, S., \& Ali, G. (2018). The relationship between fundamental analysis and stock returns based on the panel data analysis, evidence from Karachi Stock Exchange (KSE). Research Journal of Finance and Accounting, 9(3), 84-96.

Ou, J. A., \& Penman, S. H. (1989). Financial statement analysis and the prediction of stock returns. Journal of Accounting and Economics, 11(4), 295-329.

Paleari, S., \& Redondi, R. (2005). Regulation effects on company beta components. Bulletin of Economic Research, 57(4), 317-346.

Pamplona, N., \& Wiziack, J. (2017). Governo propõe privatização da Eletrobras. Recuperado em 11 janeiro, 2018 de http://www1.folha.uol.com.br/mercado/2017/08/1911776-governo-propoeprivatizacao-da-eletrobras.shtml

Peltzman, S. (1976). Toward a more general Theory of Regulation. Journal of Law and Economics, 19(2), 211-240.

Petcharabul, P., \& Romprasert, S. (2014). Technology Industry on Financial Ratios and Stock Returns. Journal of Business and Economics, 5(5), 739-746.

Pinheiro, J. L. (2016). Mercado de capitais (8a ed.). São Paulo: Atlas.

Pohlmann, M. C., \& Alves, F. J. S. Regulamentação. (2012). In A. B. Lopes \& S. Iudícibus (Coord.). Teoria avançada da contabilidade (2a ed., pp. 235-275). São Paulo: Atlas.

Posner, R. A. (1974). Theories of Economic Regulation. The Bell Journal of Economics and Management Science, 5(2), 335-358.

Prado, F. A. de A., Jr., \& Silva, A. L. R. da (2013). Sobre reformas e concessões no setor elétrico brasileiro: uma análise crítica. Revista Estratégica, 11(2), 85-115. 
Riantani, S., Hartaya, T., \& Hasanah, A. (2011). Analysis of debt to equity ratio and return on assets and its effect to closing price of the mining industry listed in BEI. ASEAN Conference Scientific and Social Science Research, Universiti Teknologi Mara, Malaysia.

Salgado, L. H. (2003) Agências regulatórias na experiência brasileira: um panorama do atual desenho institucional. Texto para discussão n. ${ }^{\circ}$ 941. Rio de Janeiro: IPEA.

Santos, V. A. dos (2017). Um modelo de análise fundamentalista de ações de instituições financeiras brasileiras. Dissertação de Mestrado em Ciências Contábeis, Universidade de Brasília, Brasília, DF, Brasil.

Schymura, L. G. (2014). Regulação e aspectos institucionais brasileiros. In S. Guerra (Org.). Regulação no Brasil: uma visão multidisciplinar (pp. 261-274). Rio de Janeiro: Editora FGV.

Sharma, M., \& Sharma, P. (2009). Prediction of stock returns for growth firms - a fundamental analysis. Vision: The Journal of Business Perspective, 13(3), 31-40.

Smith, R. T., Bradley, M., \& Jarrell, G. (1986). Studying firm-specific effects of regulation with stock market data: an application to oil price regulation. The RAND Journal of Economics, 17(4), 467-489.

Stigler, G. J. (1971). The Theory of Economic Regulation. The Bell Journal of Economics and Management Science, 2(1), 3-21.

Su, D., \& Fleisher, B. M. (1998). Risk, return and regulation in chinese stock markets. Journal of Economics and Business, 50(3), 239-256.

Taani, K., \& Banykhaled, M. H. H. (2011). The effect of financial ratios, firm size and cash flows from operating activities on earnings per share: (an applied study: on Jordanian Industrial Sector). International Journal of Social Sciences and Humanity Studies, 3(1), 197-205.

Taffarel, M., Silva, W. V. da, \& Clemente, A. (2013). Risco regulatório e reação do mercado: análise do setor de energia elétrica brasileiro. Revista Universo Contábil, 9(1), 121-134.

Wooldridge, J. M. (2016). Introductory econometrics: a modern appoach (6a ed.). Boston: Cengage Learning.

Zeitun, R., \& Tian, G. G. (2007). Capital structure and corporate performance: evidence from Jordan. Australasian Accounting, Business and Finance Journal, 1(4), 1-24. 